\title{
THE LIMIT SETS OF QUASIFUCHSIAN PUNCTURED SURFACE GROUPS AND THE TEICHMÜLLER DISTANCES
}

\author{
HideKI MiYACHI
}

To the memory of Professor Nobuyuki Suita

\section{Introduction}

Let $S$ be the interior of a compact surface with negative Euler characteristic. By virtue of Bers' simultaneous uniformization (cf. [5]), a triple $(X, h, Y)$, which consists of two hyperbolic surfaces $X$ and $Y$ of finite area homeomorphic to $S$ and a homeomorphism $h: X \rightarrow Y$, determines a unique quasifuchsian group $G$ isomorphic to $\pi_{1}(S)$ up to conjugation by Möbius transformations. Since $G$ acts discontinuously on the upper-half 3 -space $\mathbf{H}^{3}$, one may expect that the relative location between marked surfaces of the boundary at infinity of $M_{G}:=\mathbf{H}^{3} / G$ in the Teichmüller space of $S$ could be analyzed by intrinsic information from the geometry of $M_{G}$. Indeed, J. Brock ([6]) observed that the Weil-Petersson distance between marked surfaces at infinity is comparable with the volume of the convex core of $M_{G}$.

Our main theorem in this paper is to give an estimate for the Teichmüller distance between marked surface at infinity via complex lengths of geodesics in the quotient manifold of $G$. It is known that an appropriate collection of complex lengths of elements in $G$ determines the geometry of $M_{G}$, and visa versa (e.g. [13]).

This paper is organized as follows: Our main theorem will be stated in $\S 3$. We will recall in $\S 4$ the width formula for the limit set of quasifuchsian punctured surface groups by Hirotaka Akiyoshi, Makoto Sakuma and the author ([3] and [4]). Our width formula plays a crucial role to obtain our estimates (3.1) and (3.2) below. In $\S 5$, we will prove our main theorem. Furthermore, we give a distortion lemma for equivariant quasiconformal mappings in proving our results.

CONVENTION. Throughout this paper, all (hyperbolic) surfaces are of analytically finite type and have at least one puncture and we use the symbol $S$ to represent the interior of a compact surface with negative Euler characteristic. Furthermore, we fix a hyperbolic structure of finite area on $S$.

Received February 25, 2004; revised November 2, 2004. 
Acknowledgements. The author wishes to thank Osaka University for financial support and to Dr. Hirotaka Akiyoshi, Prof. Ken'ichi Ohshika, and Prof. Makoto Sakuma for giving him fascinating mathematical ideas and conversations.

$\mathrm{He}$ also expresses his thanks to Prof. Toshiyuki Sugawa for telling him a paper of Agard and Gehring [1] and to Prof. Masahiko Taniguchi, Prof. Yoichi Imayoshi, and Prof. Ken-ichi Sakan for useful comments and their long-time encouragements.

Finally, he thanks the referee for his/her valuable comments.

\section{Notation}

We shall give some notations.

\subsection{Teichmüller spaces and Teichmüller distances}

The Teichmüller space Teich $(S)$ of $S$ is the space of equivalence classes of pairs $(X, f)$, we call it a marked surface, of a hyperbolic surface $X$ and a quasiconformal mapping $f: S \rightarrow X$. Two marked surfaces $(X, f)$ and $(Y, g)$ are equivalent if there is an isometry $h: X \rightarrow Y$ such that $h \circ f$ is homotopic to $g$ on $S$. We abbreviate $(X, f)$ by $X$ when its marking $f$ is not essentially used in the context.

The Teichmüller distance $d_{T}$ on $\operatorname{Teich}(S)$ is defined by

$$
d_{T}((X, f),(Y, g))=\inf _{h} \log K(h)
$$

where $h$ runs over all quasiconformal mappings from $X$ to $Y$ which are homotopic to $g \circ f^{-1}$ and $K(h)$ denotes the maximal dilatation of $h$ (see [10]).

\subsection{Quasifuchsian groups and Bers' uniformization}

A quasifuchsian group is a finitely generated Kleinian group given by a quasiconformal deformation of a Fuchsian group of the first kind. By definition, any quasifuchsian group is isomorphic to the fundamental group of a compact surface and its limit set is a quasicircle.

Let $X$ and $Y^{*}$ be hyperbolic surfaces with mutually different orientations and $h^{*}: X \rightarrow Y^{*}$ an orientation reversing homeomorphism. L. Bers ([5]) showed that the triple $\left(X, h^{*}, Y^{*}\right)$ determines a unique quasifuchsian group $G$ (up to conjugation) such that the boundary at infinity of $M_{G}=\mathbf{H}^{3} / G$ is isometric to $X \cup Y^{*}$ and $h^{*}$ induces a quasiconformal reflection with respect to the limit set of $G$ which is commutative with each element of $G$. Let $Y$ be the mirror image of $Y^{*}$ and $h: X \rightarrow Y$ the orientation preserving homeomorphism induced by $h^{*}$. In this paper, we say that $G$ is uniformized by a triple $(X, h, Y)$.

A quasifuchsian group $G$ is said to be uniformized by $(X, f),(Y, g) \in$ $\operatorname{Teich}(S)$ if $G$ is uniformized by $\left(X, g \circ f^{-1}, Y\right)$. In this case, a quasiconformal mapping $f: S \rightarrow X$ induces a quasifuchsian representation $\rho$ of $\pi_{1}(S)$ to $\operatorname{PSL}_{2}(\mathbf{C})$ such that $G=\rho\left(\pi_{1}(S)\right)$. We also say that $\rho$ is uniformized by $(X, f)$ and $(Y, g)$. 


\subsection{Complex lengths and Bending laminations}

Let $\rho: \pi_{1}(S) \rightarrow \operatorname{PSL}_{2}(\mathbf{C})$ be a representation. Take a $\gamma \in \pi_{1}(S)$ so that $\rho(\gamma)$ is loxodromic. We let $\lambda_{\rho}(\gamma)(\in \mathbf{C} / 2 \pi \mathbf{Z})$ denote the complex length associated with $\gamma$, that is, $\operatorname{Re} \lambda_{\rho}(\gamma)$ is the hyperbolic length of the geodesic in $M=\mathbf{H}^{3} / \rho\left(\pi_{1}(S)\right)$ corresponding to $\gamma$ and $\operatorname{Im} \lambda_{\rho}(\gamma)$ is the angle through which a normal vector turns when parallely translated once around the curve. The complex length satisfies the equation

$$
\operatorname{tr}^{2} \rho(\gamma)=4 \cosh ^{2} \frac{\lambda_{\rho}(\gamma)}{2}
$$

Let $\mathscr{S}(S)$ be the set of free homotopy classes of non-peripheral and nontrivial simple closed curves on $S$. Since $\lambda_{\rho}\left(\alpha \gamma \alpha^{-1}\right)=\lambda_{\rho}(\gamma)$ for $\alpha, \gamma \in \pi_{1}(S)$, by abuse of notation, we denote $\lambda_{\rho}(\gamma)$ for $\gamma \in \mathscr{S}(S)$ to represent the complex length of an element in the conjugacy class. For $\gamma \in \mathscr{S}(S)$, from (2.1), the suitable lift of $\lambda_{\rho}(\gamma)$ (we use the same symbol to represent the lift, for simplicity) is a holomorphic function on the quasifuchsian space which takes real values on the Fuchsian slice (see [9]).

Suppose $\rho: \pi_{1}(S) \rightarrow \mathrm{PSL}_{2}(\mathbf{C})$ to be a quasifuchsian representation. If $\rho\left(\pi_{1}(S)\right)$ is not Fuchsian, the boundary of the convex core $M_{0}$ of $M$ has two components $\partial^{ \pm} M_{0}$. Each component is a complete hyperbolic surface bent along a measured geodesic lamination. The pull-backs of the measured geodesic laminations on $\partial^{ \pm} M_{0}$ via $\rho$ define measured geodesic laminations $p l^{ \pm}$on $S$, which are called the bending laminations of $\rho$ (see [7] and [9]). Let us denote by $\mathscr{P} \mathscr{M L}(S)$ the projective measured lamination space of $S$. Taking the geodesic representatives, we recognize any element $\gamma \in \mathscr{S}(S)$ as a geodesic lamination. Furthermore, $\gamma \in \mathscr{S}(S)$ admits a canonical transversal measure that assigns the intersection number with the geodesic representative of $\gamma$ to any transversal arc. Thus $\mathscr{S}(S)$ is canonically identified with a subset of $\mathscr{P} \mathscr{M} \mathscr{L}(S)$.

\section{Results}

This section gives our main theorems. First we begin with the case of once punctured tori (Theorem 1), and then we deal with the general case (Theorem 2). Of course, the former case follows from the latter (cf. [4]). However, the former case will be helpful for readers to understand the estimates to originate from the intrinsic geometry of corresponding quasifuchsian manifold.

\subsection{Case of once punctured tori}

Assume $S$ to be a once punctured torus. In this case, $\mathscr{P} \mathscr{M L}(S)$ is identified with $S^{1}=\mathbf{R} \cup\{\infty\}$ and $\mathscr{S}(S)$ is regarded as the set of rational points in $\mathscr{P} \mathscr{M L}(S)$. Let $\left[p l^{ \pm}\right]$be the projective classes of the bending laminations of a quasifuchsian representation $\rho$ of $\pi_{1}(S)$. Since $\left[p l^{+}\right] \neq\left[p l^{-}\right]$(see $\left.[9]\right), \mathscr{P} \mathscr{M L}(S)-$ $\left\{\left[p l^{-}\right],\left[p l^{+}\right]\right\}$consists of two intervals in $\mathscr{P} \mathscr{M L}(S)$. This induces the decomposition $\mathscr{S}(S)-\left\{\left[p l^{-}\right],\left[p l^{+}\right]\right\}=\mathscr{S}_{L} \cup \mathscr{S}_{R}$. 
THEOREM 1. Let $\rho$ be the quasifuchsian representation uniformized by two marked surfaces $X, Y \in \operatorname{Teich}(S)$. Then the Teichmüller distance $d_{T}(X, Y)$ between $X$ and $Y$ satisfies

$$
d_{T}(X, Y) \geq \log \kappa(|w(\rho)|)
$$

where

$$
\begin{aligned}
& w(\rho)=2 \operatorname{Im} \sum_{\gamma \in \mathscr{S}_{R}} \frac{1}{1+e^{\lambda_{p}(\gamma)}}, \\
& \kappa(y)=\frac{2}{\pi} \mu\left(\frac{e^{-\pi y / 2}}{\sqrt{1+e^{-\pi y}}}\right)
\end{aligned}
$$

and $\mu(r)$ is the modulus of the unit disk slit along the real axis from 0 to $r$.

\subsection{Case of punctured surfaces}

Suppose $S$ to be a general punctured surface. In this section, we fix a puncture $p$ of $S$ and uniformize $S$ in such a way that one of the corresponding primitive elements to the puncture $p$ is a parallel translation $z \mapsto z+1$ in the deck transformation group of the universal covering space $\mathbf{H}^{2} \rightarrow S$.

A simple $\operatorname{arc} \delta$ in $S$ with both ends in the puncture $p$ is said to be essential if it does not bound a monogon (i.e., a disk with one point removed from its boundary). By $\Delta$ (resp. $\vec{\Delta}$ ) we denote the set of the isotopy classes of unoriented (resp. oriented) essential simple arcs in $S$ with both ends in $p$. We shall abuse notation to denote a simple loop or an arc and its isotopy class by the same symbol. For each essential arc $\delta \in \Delta$ (or $\delta \in \vec{\Delta}$ ) there is a unique (up to isotopy) pair of simple loops $\alpha(\delta)$ and $\beta(\delta)$ such that $\alpha(\delta) \cup \beta(\delta)$ bounds a punctured annulus containing $\delta$ (cf. Proposition 1 of [12]). These loops determine a pair of elements of $\mathscr{S}(S) \cup \mathscr{P}(S)$, where $\mathscr{P}(S)$ is the set of the isotopy classes of peripheral simple loops in $S$. We note the following facts.

1. If $S$ is a once punctured torus, then $\alpha(\delta)=\beta(\delta) \in \mathscr{S}(S)$. Otherwise, $\alpha(\delta) \neq \beta(\delta)$.

2. One of $\alpha(\delta)$ and $\beta(\delta)$ belongs to $\mathscr{P}(S)$ if and only if $\delta$ bounds a oncepunctured monogon.

Let $\mathscr{G}$ be the set of oriented complete simple geodesics in the hyperbolic surface $S$ emanating from the puncture $p$. Then the set $\vec{\Delta}$ is regarded as a subset of $\mathscr{G}$. Let $\tilde{\mathscr{G}}$ be the set of oriented complete geodesics in $\mathbf{H}^{2}$ emanating from $\infty$ which projects to a simple geodesic in $S$. Then $\tilde{\mathscr{G}}$ is identified with a subset of $\mathbf{R}=\partial \mathbf{H}^{2}-\{\infty\}$ by associating each element $\tilde{\mu} \in \tilde{\mathscr{G}}$ with its endpoint. This induces an identification of $\mathscr{G}$ with a subset of the circle $S_{p}^{1}:=\mathbf{R} /\langle z \mapsto z+1\rangle=$ $\mathbf{R} / \mathbf{Z}$.

Let $\rho$ be a quasifuchsian (not Fuchsian) representation, and let $\left|p l^{ \pm}\right|$be the underlying geodesic lamination of the bending laminations $p l^{ \pm}$of $\rho$. Then $\left|p l^{ \pm}\right|$is disjoint from a neighborhood of $p$, and hence we can find, for each $\varepsilon= \pm$, a complete simple geodesic $\mu^{\varepsilon} \in \mathscr{G}$ which is disjoint from $\left|p l^{\varepsilon}\right|$. We have $\mu^{-} \neq \mu^{+}$, because $\left.\mid p l^{+}\right\rfloor \neq\left|p l^{-}\right|$. Since $\vec{\Delta}$ is identified with a subset of $S_{p}^{1}$, we obtain a partition of $\vec{\Delta}-\left\{\mu^{-}, \mu^{+}\right\}$into two subsets, $\vec{\Delta}_{L}$ and $\vec{\Delta}_{R}$. 
THEOREM 2. Let $\rho$ be a quasifuchsian representation of $\pi_{1}(S)$ uniformized by $X, Y \in \operatorname{Teich}(S)$. Fix a puncture $p$ of $S$ and let $\vec{\Delta}_{L}, \alpha(\delta)$, and $\beta(\delta)\left(\delta \in \vec{\Delta}_{L}\right)$ be as above. Then the Teichmüller distance between $X$ and $Y$ satisfies

$$
d_{T}(X, Y) \geq \log \kappa(|w(\rho)|),
$$

where

$$
w(\rho)=2 \operatorname{Im} \sum_{\delta \in \vec{\Delta}_{L}} \frac{1}{1+e^{1 / 2\left(\lambda_{p}(\alpha(\delta))+\lambda_{p}(\beta(\delta))\right)}},
$$

and $\kappa$ is the function in Theorem 1.

\subsection{Remarks}

We give three remarks concerning our estimates (3.1) and (3.2).

(1) The function $\mu(r)$ is strictly decreasing and expressed exactly as follows (cf. p. 60 of [11]).

$$
\mu(r)=\frac{\pi \mathscr{K}\left(\sqrt{1-r^{2}}\right)}{2 \mathscr{K}(r)} \quad(0<r<1),
$$

where $\mathscr{K}(r)$ is the complete elliptic integral of the first kind:

$$
\mathscr{K}(r)=\int_{0}^{1} \frac{d t}{\sqrt{\left(1-t^{2}\right)\left(1-r^{2} t^{2}\right)}} .
$$

(2) The function $\kappa$ is strictly increasing. Furthermore, since $\lim _{r \rightarrow 0}(\mu(r)-$ $\log (4 / r))=0$ (cf. (2.11) of p. 62 in [11]), $\kappa$ behaves asymptotically as

$$
\kappa(y)-\left(\frac{4}{\pi} \log 2+y\right)=o(1)
$$

when $y \rightarrow+\infty$.

(3) Unfortunately, it seems to exist no upper bound of the Teichmüller distance by using $w(\rho)$. Indeed, one can check that $w(\rho)$ remains bounded when $\rho$ is a geometrically finite punctured torus group (cf. [3]).

\section{Widths of the limit sets}

In this section, we recall formulas for widths of the limit sets of quasifuchsian punctured groups given in [3] and [4].

\subsection{Widths of Jordan curves}

Let $\Lambda$ be a Jordan curve in $\hat{\mathbf{C}}$ which is invariant under the action of a parallel translation $z \mapsto z+2$. The width width $(\Lambda)$ of $\Lambda$ is defined to be

$$
\operatorname{width}(\Lambda)=m^{+}(\Lambda)-m^{-}(\Lambda)
$$




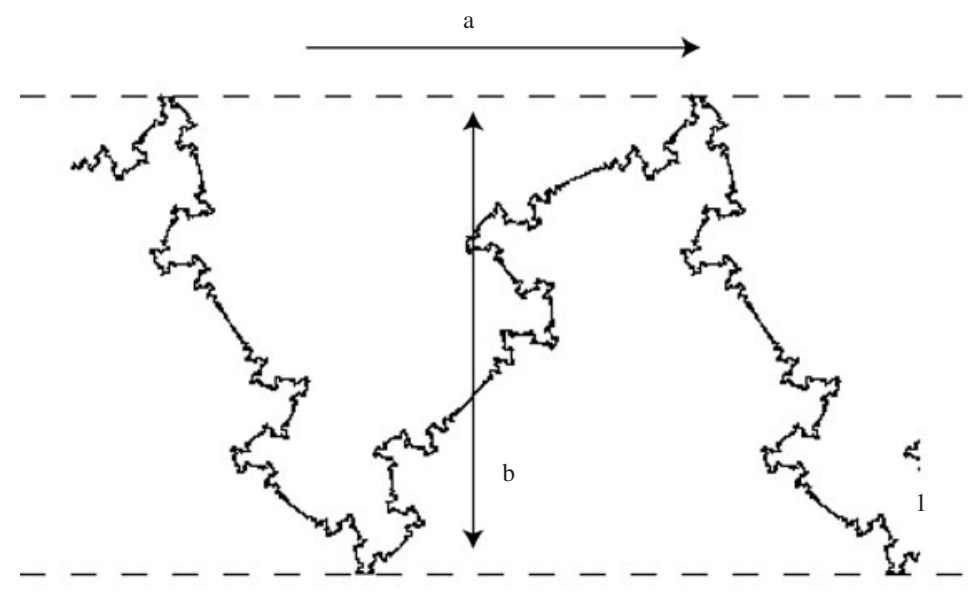

$\underline{\text { PSfrag replacements }}$

$$
\begin{array}{r}
z \mapsto z+2 \\
2 \text { width }(\Lambda)
\end{array}
$$

FiguRE 1. The width of a quasicircle. This picture was drawn by using a computer program "OPTi" created by Professor Masaaki Wada.

where $m^{+}(\Lambda):=\max \{\operatorname{Im} z \mid z \in \Lambda \cap \mathbf{C}\}$ and $m^{-}(\Lambda):=\min \{\operatorname{Im} z \mid z \in \Lambda \cap \mathbf{C}\} \quad$ (see Figure 1).

\subsection{Width formula}

The following is established in [3] and [4].

THEOREM 3. Let $p$ be a puncture of $S$ and $\rho$ a quasifuchsian representation of $\pi_{1}(S)$ normalized so that one of the corresponding primitive elements to $p$ is $z \mapsto z+2$. With the notation as in Theorems 1 and 2 , the width $\operatorname{width}(\Lambda)$ of the limit set $\Lambda$ of $\rho\left(\pi_{1}(S)\right)$ is equal to $|w(\rho)|$.

\section{Proof of Theorem}

We begin with stating the following proposition.

Proposition 1. Let $F$ be a $K$-quasiconformal mapping equivariant with respect to the action of $z \mapsto z+2$, that is, $F$ satisfies $F(z+2)=F(z)+2$, and $\Lambda$ denote its image of $\mathbf{R} \cup\{\infty\}$. Then

$$
K \geq \kappa(\operatorname{width}(\Lambda)),
$$

where $\kappa$ is the function defined in Theorem 1. 
Before proving Proposition 1 we shall deduce our main theorems from this proposition and Theorem 3. Indeed, let $\rho$ be a quasifuchsian representation of $\pi_{1}(S)$ uniformized by two marked surfaces $X$ and $Y$. Since $\kappa(0)=1$ and hence (3.1) and (3.2) are trivial for any Fuchsian representation, we may assume that $\rho$ is not Fuchsian.

Let $K$ be the maximal dilatation of a quasiconformal mapping from $X$ to $Y$ preserving their markings. Suppose that a primitive element corresponding to $p$ via $\rho$ is $z \mapsto z+2$. Then, by the argument similar to that by L. Bers in [5], we see that $\Lambda$ is the image of a $K$-quasiconformal mapping $F$ of $\mathbf{R} \cup\{\infty\}$ with $F(z+2)=F(z)+2$. Therefore, by Proposition 1 and Theorem 3, we conclude the assertion.

\subsection{Distortion property of equivariant quasicircles}

In this section, we give a distortion lemma for equivariant quasicircles.

Proposition 2. Let $\Lambda$ be the image of $\mathbf{R} \cup\{\infty\}$ under a $K$-quasiconformal mapping equivariant with respect to the action of $z \mapsto z+2$, then for $\zeta_{1}, \zeta_{2} \in \Lambda$,

$$
\left|\operatorname{Im}\left(\zeta_{1}-\zeta_{2}\right)\right| \leq \frac{1}{\pi} \log \left(\left(\mu^{-1}(\pi K / 2)\right)^{-2}-1\right) .
$$

Furthermore, the inequality is sharp.

Remark 1. The right-hand side of the inequality (5.1) coincides with the inverse function of $\kappa$ in Theorem 1.

Proof of Proposition 2. Let $F$ be a $K$-quasiconformal mapping equivariant under the action of $z \mapsto z+2$ and set $\Lambda=F(\mathbf{R} \cup\{\infty\})$. Let $\zeta_{1}, \zeta_{2} \in \Lambda$. Without loss of generality, we may assume that $\operatorname{Im}\left(\zeta_{2}-\zeta_{1}\right) \geq 0$.

Set $G(z)=F\left(z-F^{-1}\left(\zeta_{1}\right)\right)-\zeta_{1}$ and let $x_{2}=F^{-1}\left(\zeta_{2}\right)+F^{-1}\left(\zeta_{1}\right)$. Then $G\left(x_{2}\right)=\zeta_{2}-\zeta_{1}$. From the equivariance of $G$ under $z \mapsto z+2$, there exists a $K$ quasiconformal mapping $H$ of $\mathbf{C}$ such that $H(0)=0, H(1)=1$ and $H \circ \operatorname{proj}=$ $\operatorname{proj} \circ G$ where $\operatorname{proj}(z)=e^{\pi i z}$. It follows from Lemmas 5.1 and 5.16 of [15] and Teichmüller's theorem (cf. e.g. [1] or [2]) that

$$
\begin{aligned}
\frac{1}{2} \log K & \geq d\left(e^{\pi i x_{2}}, H\left(e^{\pi i x_{2}}\right)\right) \\
& \geq d\left(-1,-e^{-\pi \operatorname{Im}\left(\zeta_{2}-\zeta_{1}\right)}\right)=\left|\Phi\left(e^{-\pi\left|\operatorname{Im}\left(\zeta_{2}-\zeta_{1}\right)\right|}\right)\right|,
\end{aligned}
$$

where $d$ is the hyperbolic distance on $\mathbf{C}-\{0,1\}$ of constant curvature -4 , and

$$
\Phi(x)=\frac{1}{2} \log \frac{\mathscr{K}\left(x^{1 / 2}(1+x)^{-1 / 2}\right)}{\mathscr{K}\left((1+x)^{-1 / 2}\right)}=\frac{1}{2} \log \frac{2}{\pi} \mu\left((1+x)^{-1 / 2}\right)
$$

for $x>0$. Since $0<\mu(r) \leq \pi / 2$ for $r \geq 1 / \sqrt{2}$, we conclude that

$$
\mu\left(\left(1+e^{-\pi\left|\operatorname{Im}\left(\zeta_{2}-\zeta_{1}\right)\right|}\right)^{-1 / 2}\right) \geq \frac{\pi}{2 K} .
$$


Together with the equation $\mu(r) \cdot \mu\left(\left(1-r^{2}\right)^{1 / 2}\right)=\pi^{2} / 4$ (cf. (2.7) of p. 61 in [11]), we deduce the assertion. The equality in (5.1) is attained by a lift (with respect to proj) of a $K$-quasiconformal mapping which fixes 0,1 and $\infty$, and maps -1 to $-x_{K}<0$ with $d\left(-1,-x_{K}\right)=(\log K) / 2$ (cf. Lemma 3 of [1]).

\subsection{Proof of Proposition 1}

Since $\Lambda$ is invariant under the action of $z \mapsto z+2$, we can find $\zeta_{1}, \zeta_{2} \in \Lambda$ such that $\operatorname{Im}\left(\zeta_{1}\right)=m^{-}(\Lambda)$ and $\operatorname{Im}\left(\zeta_{2}\right)=m^{+}(\Lambda)$. Then by Proposition 2, we have

$$
\begin{aligned}
\operatorname{width}(\Lambda) & =\operatorname{Im}\left(\zeta_{2}-\zeta_{1}\right) \\
& \leq \frac{1}{\pi} \log \left(\left(\mu^{-1}(\pi K / 2)\right)^{-2}-1\right) \\
& =\kappa^{-1}(K)
\end{aligned}
$$

Since $\kappa$ is strictly increasing, we complete the proof of Proposition 1 .

\section{REFERENCES}

[1] S. B. Agard and F. W. Gehring, Angles and Quasiconformal mappings, Proc. London Math. Soc. (3) 14A (1965), 1-21.

[2] L. V. Ahlfors, Lecture on quasiconformal mappings, Van Nostrand. Princeton, New Jersey, 1966.

[3] H. Akiyoshi, H. Miyachi and M. Sakuma, A refinement of McShane's identity of once punctured torus groups, in In the tradition of Ahlfors and Bers, III; Contemporary Math. 355 (2004), 21-40.

[ 4 ] H. Akiyoshi, H. Miyachi and M. Sakuma, Variations of McShane's identity for punctured surface groups, to appear in the Proceedings of the conference "Spaces of Kleinian groups and hyperbolic gemetry" held at the Newton Institute, 2003.

[5] L. Bers, Simultaneous uniformization, Bull. Amer. Math. Soc. 66 (1960), 94-97.

[6] J. BRock, The Weil-Petersson metric and volumes of 3-dimensional hyperbolic convex cores, Jour. Amer. Math. Soc. 16 (2003), 495-535.

[7] D. B. A. Epstein And A. Marden, Convex hulls in hyperbolic space, a theorem of Sullivan, and measured pleated surfaces, Analytical and geometric aspects of hyperbolic space (Coventry/Durham, 1984), London Math. Soc. Lecture Note Ser. 111, Cambridge Univ. Press, Cambridge, 1987, 113-253.

[ 8 ] F. W. Gehring, Definitions for a class of plane quasiconformal mappings, Nagoya Math. J. 29 (1967), 175-184.

[9] L. KeEn AND C. SERIES, Pleating invariants for punctured torus groups, Topology 43 (2004), 447-491.

[10] Y. Imayoshi AND M. TANIGUChI, An introduction to Teichmüller spaces, Springer-Verlag, Tokyo, 1992.

[11] O. Lehto AND K. I. Virtanen, Quasiconformal mappings in the plane, Springer, Berlin 1973.

[12] G. McShane, Simple geodesics and a series constant over Teichmuller space, Invent. Math. 132 (1998), 607-632.

[13] Y. Minsky, The classification of punctured-torus groups, Ann. of Math. 149 (1999), 559-626. 
[14] Z. Nehari, Conformal mapping, McGrow-Hill Book Co., New York, 1952.

[15] T. Sugawa and M. Vuorinen, Some inequalities for the Poincaré metric on Plane domains, to appear in Math. Z.

Department of Mathematical Sciences

Ishizaka, Hatoyama, Hiki, Saitama, 350-0394

JAPAN

e-mail: miyachi@r.dendai.ac.jp 